\title{
Advanced Segmentation Algorithms for the Three- Dimensional Analysis of X-Ray CT Data from Geomaterials
}

\author{
Loes BRABANT ${ }^{1}$, Jelle VLASSENBROECK ${ }^{2}$, Tim DE KOCK ${ }^{3}$, Manuel DIERICK ${ }^{1}$, Veerle \\ CNUDDE $^{3}$, Luc VAN HOOREBEKE ${ }^{1}$ \\ ${ }^{1}$ Department of Physics and Astronomy, Ghent University, Faculty of Sciences, Proeftuinstraat \\ 86, 9000 Ghent, Belgium [email: Loes.Brabant@UGent.be; Manuel.Dierick@UGent.be; \\ Luc.VanHoorebeke@UGent.be] \\ 2 inCT, IIC UGent, Technologiepark 3, 9052 Ghent, Belgium \\ [email: Jelle.Vlassenbroeck@inCT.be] \\ ${ }^{3}$ Department of Geology and Soil Science, Ghent University, Faculty of Sciences, Krijgslaan \\ 281/S8, 9000 Ghent, Belgium [email: Tim.Dekock@UGent.be; Veerle.Cnudde@UGent.be]
}

\begin{abstract}
X-ray Computed Tomography (CT) is a non-destructive technique which enables the visualization of the internal structure of all kinds of samples, including geological samples. Additionally, the resulting datasets can be analyzed to obtain quantitative information. As geological samples often contain different components such as different minerals, pores and a matrix, accurate segmentation techniques are of the utmost importance in order to obtain correct results.

This paper illustrates the use of advanced segmentation algorithms applied on a Belgian glauconite-rich sandstone, the Beerlegem fieldstone. These segmentation algorithms allow extraction of the different grains in the stone, setting the stage for further analysis.
\end{abstract}

\section{Introduction}

The fieldstone is a green- to gray-colored, glauconite-bearing, silicified sandstone, with sparse silicified fossils and sedimentary structures. Essentially, this definition of the fieldstone is based on its lithology and not on its stratigraphy. Therefore it comprises stones from different stone banks with different ages, belonging to different members in the early- to middle- Eocene of Flanders. Their similarities make it different to track back their origin for archaeological purposes. The use of several techniques for provenance studies is currently investigated. In addition to using traditional microscopy and chemical research techniques, X-ray CT can be a valuable tool for 3D structural characterization of the fieldstones, in order to contribute to the provenance study of this stone.

To extract quantitative information, several software packages for the analysis of three dimensional (3D) data exist. The 'Centre for X-ray Tomography' of the Ghent University (UGCT) covers the entire X-ray tomography process from design and construction of the CT scanners to data acquisition, processing, reconstruction and visualization. To gain optimal control over the data processing a 3D-analysis software package, Morpho+, was developed in house (Vlassenbroeck et al, 2007; Brabant et al., 2011).

An important aspect in this research is the quantification of the grains of the fieldstone. Therefore these grains need to be accurately segmented. In 3D-analysis, the most commonly used al- 
gorithm for segmentation is the watershed based segmentation (Vincent and Soille, 1991). However, a drawback of this method is that it often leads to over-segmentation (i.e. more objects are separated than actually needed). Therefore an intelligent semi-automatic "smart rejoin" algorithm (Brabant et al., 2011) can be applied to obtain a more accurate segmentation of the grains. To separate different components in the stone, selection parameters can be used.

\section{Materials and Methods}

\subsection{The Beerlegem Fieldstone}

The Belgian sandstone used in this research is the Beerlegem fieldstone. It originates from the sand quarry in Beerlegem, geologically located in the Ypresian stage (Gentbrugge Formation, Vlierzele Member). These fieldstones are typically glauconiferous fine-grained quartz arenites with a siliceous cement. It contains subrounded to angular quartz grains with some quartz splinters and numerous sponge needles. The sand fraction consists mainly of monocrystalline (sometimes polycrystalline) quartz with additional glauconite, feldspar (often weathered), chert (silex) and muscovite. Some heavy minerals are also found in this stone. Most glauconite grains show some oxidation in thin section and some contain clusters of oxidized framboidal pyrite. Chalcedony cement fills the inter-particular space.

\subsection{Scanning Conditions}

The Beerlegem fieldstone was scanned with the flexible micro CT set-up from UGCT. The transmission head (FeinFocus, FXE-160.51) was selected with a tube voltage of $80 \mathrm{kV}$ and a tube current of $100 \mu \mathrm{A}$. The PerkinElmer 1620 CN3-CS (2024 x 2024 pixels) was used as the detector. 1000 projections were taken over $360^{\circ}$, with an exposure time of $2000 \mathrm{~ms}$ per frame and 2 frames per projection. For this set-up a voxel size of $4.78 \mu \mathrm{m}$ was obtained in all directions. The resulting projection images were reconstructed with the in-house developed reconstruction software package Octopus (Vlassenbroeck et al, 2007) in order to obtain the crosssection images, which can be analyzed. The resulting volume has a height of $4.16 \mathrm{~mm}$, a depth of $3.97 \mathrm{~mm}$ and a width of $4.22 \mathrm{~mm}$.

\subsection{Analysis Process}

First the original gray value images are converted into binary images. This can be done with a single threshold operation, in which the user selects one threshold gray value. All the voxels with a value below this threshold are set to zero (background); those with a larger value are set to one (foreground). The threshold can also be selected automatically using Otsu's method (Otsu, 1979). More advanced methods for thresholding are also available; however, for the objectives of this study a simple threshold is sufficient.

Subsequently the connected components of foreground voxels in the obtained binary image are detected and color labeled. Often a threshold operation alone is not sufficient to obtain the desired objects, and additional segmentation is needed. Grains which are in reality separated from one another appear to be connected, due to limited resolution of the scan. Additional segmentation is thus necessary.

The watershed based separation (Vincent and Soille, 1991) performs an additional segmentation. As input the Euclidean distance transform of the binary image or the gradient of the original image can be used. The Euclidean distance transform determines for each voxel the closest distance to the border; to calculate it, the Voronoi diagram is used (Guan and Ma, 1998). For the Beerlegem fieldstone the Euclidean distance transform was determined. 


\subsection{Smart Rejoin}

When a watershed based separation induces over-segmentation, a rejoin operation is necessary. It is possible to manually rejoin selected objects, however this is a time-consuming job (especially for large datasets) and mistakes are easily made. Therefore a semi-automatic method to rejoin was developed at UGCT and added to Morpho+ (Brabant et al., 2011).

This "smart rejoin" method evaluates the separations that are introduced when performing a watershed based separation. For each separation the maximum opening - this is the diameter of the largest inscribed sphere - of the two objects it separates, is determined. The largest of these $\left(d_{o b j}\right)$ is then compared with the diameter of the maximum inscribed circle in the separation $\left(d_{s \otimes p}\right)$. Two objects are rejoined if the following equation is valid:

$$
a_{s \theta p}>\left(l x a_{o b j}\right)
$$

With $d$ a user specified level between 0 and 1 . Thus, objects are rejoined if their separation is not sufficiently smaller than their cross-section. More information about this method can be found in (Brabant et al., 2011).

\subsection{Component Classification}

As mentioned above, the Beerlegem fieldstone contains various components. For this study we are interested in the quartz and the glauconite grains. In order to make a distinction between the different components "selection parameters" can be used. These parameters need to have different values for the different components.

The glauconite grains can easily be identified; as they have a higher gray value in the images. Morpho+ allows selecting objects based on all kinds of parameters, including the average gray value of the object, so the glauconite grains can be selected in this way.

The distinction between the quartz grains and the cement is not straightforward as they have the same gray value in the images. However, the maximum opening of the objects belonging to the cement will be smaller than that of the grain objects. Therefore the maximum opening can be chosen as a selection parameter. When using the maximum opening as a selection parameter, the smart rejoin operation needs to be performed first to avoid erroneous classification.

When using selection parameters for segmentation one can never be sure that all the objects are classified correctly. In most cases a more severe threshold value is selected to limit the number of misclassified objects, even though this means that some objects of the correct component will not be taken into account for the analysis.

\section{Results and Discussion}

Figure 1 illustrates the effects of the different segmentation algorithms. The algorithms operate in $3 \mathrm{D}$ but the result is shown in 2D for demonstration purposes. In Figure 1a, a reconstructed cross-section of the Beerlegem fieldstone is shown, figure $1 \mathrm{~b}$ shows the same cross-section after watershed based separation and figure $1 \mathrm{c}$ shows this cross-section after smart rejoin with $1=0.85$ . When comparing figure a with figure $1 \mathrm{~b}$ it is clear that the grains in the white regions are oversegmented. However, after smart rejoin in figure $1 \mathrm{c}$, these grains are correctly segmented. 

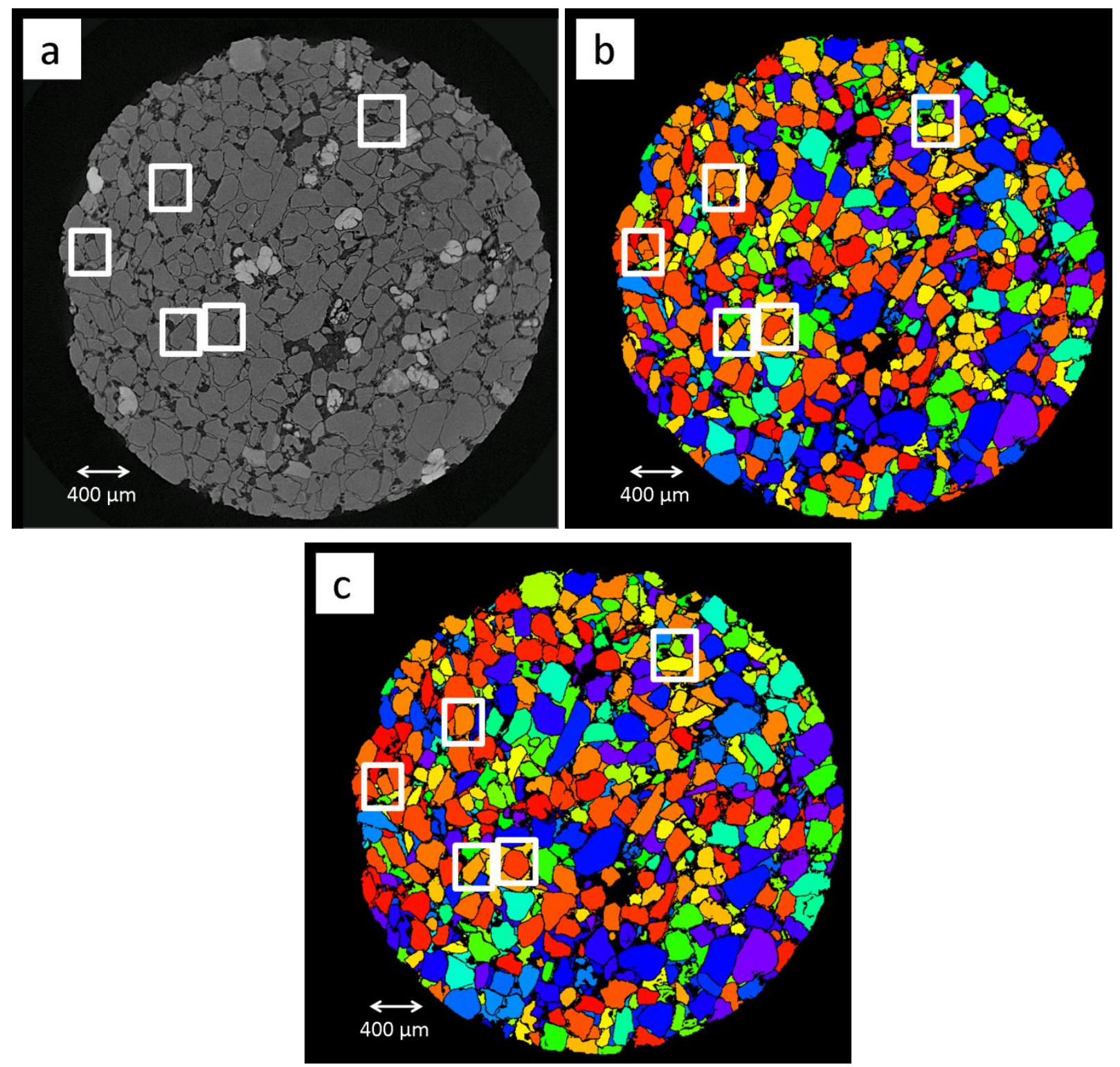

Figure 1: Reconstructed cross-section of a Beerlegem fieldstone (a), segmentation of the grains after watershed based separation (b) and smart rejoin (c). The white regions indicate the grains which are over-segmented when only the watershed based separation is performed, and which are correctly segmented after performing a smart rejoin operation

Figure 2 illustrates the segmentation of the glauconite and the quartz grains. The algorithms operate in $3 \mathrm{D}$ but the result is shown in $2 \mathrm{D}$ for demonstration purposes. The glauconite grains (Figure 2b) are selected using a strong gray value threshold - only objects with an average gray value above this threshold are included - to ensure that only the glauconite grains are included. To select the quartz grain, first a less severe gray value threshold is used to exclude glauconite and other components. Subsequently a size threshold is selected; only objects with a maximum opening above $75 \mu \mathrm{m}$ are included. In this way, the objects belonging to the cement are excluded. 

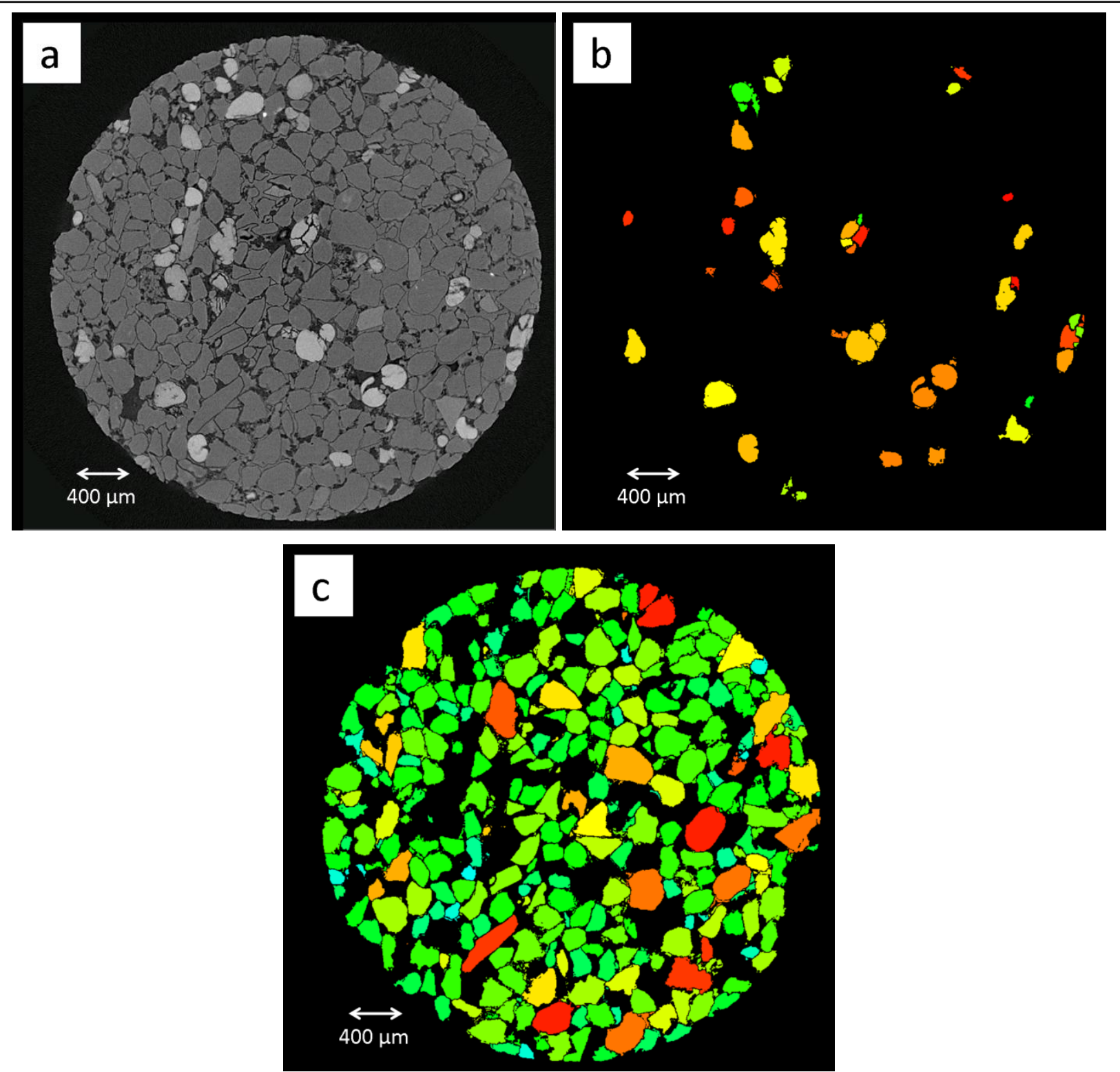

Figure 2: Reconstructed cross-section of a Beerlegem fieldstone (a), segmentation of the glauconite grains based on average gray value of the objects (b) segmentation of the quartz grains after elimination of the glauconite grains and other components based on average gray value and after elimination of the cement based on the maximum opening of the objects (c).

Once the grains are segmented, they can be quantified. Several parameters for quantification exist, such as: the surface, the maximum opening, the minimum closing, which is the diameter of the minimum circumscribed sphere, the equivalent diameter, which is the diameter of the sphere that can be created from the total number of voxels of the objects. In addition to size parameters it is possible to determine the sphericity and the orientation of each object. The sphericity is defined as the ratio of the maximum opening and the minimum closing. This will give a value between zero and one, if the sphericity is equal to one the object is a sphere; if it is close to zero the object does not resemble a sphere at all. The equivalent diameter, the maximum opening and the sphericity were determined for the grains in the Beerlegem fieldstone.

The analyzed quartz grains have a sphericity of $0.60 \pm 0.07$ whereas the glauconite grains have an average sphericity of $0.57 \pm 0.09$; the difference is thus very small. The quartz grains have an average maximum opening of $126( \pm 31) \mu \mathrm{m}$ (although the real value will be lower because the grains with a maximum opening smaller than $75 \mu \mathrm{m}$ were excluded to eliminate objects part of 
the cement) and an average equivalent diameter of $210( \pm 49) \mu \mathrm{m}$; the glauconite grains have an average maximum opening of $93( \pm 35) \mu \mathrm{m}$ and an average equivalent diameter of $164( \pm 63) \mu \mathrm{m}$. The distribution of the equivalent diameter is illustrated in Figure 3. After segmentation, the fraction of the components was determined: $73 \%$ of the material in the stone consists of quartz grains, whereas only $7 \%$ are glauconite grains. Therefore the fraction of grains with a certain size was determined instead of the total number of grains. It is clear that the fraction of quartz grains with a higher equivalent diameter is larger than that of the glauconite grains.

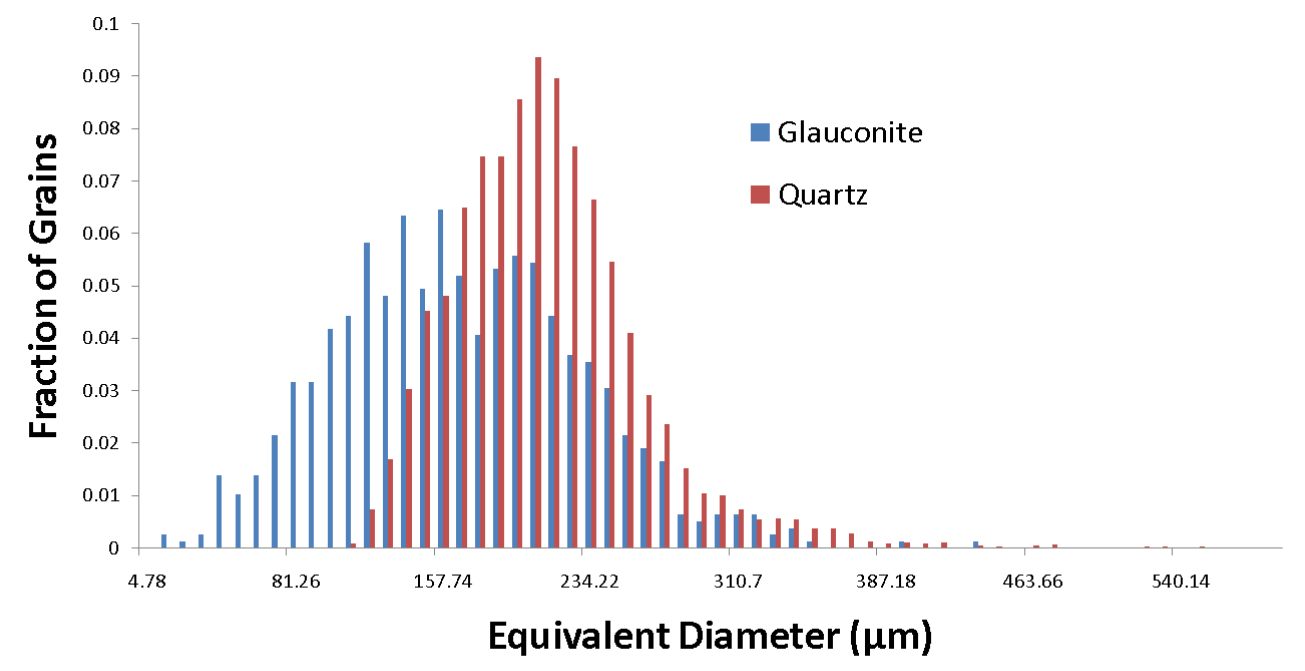

Figure 3: Distribution of the equivalent diameter of the glauconite and the quartz grains in the Beerlegem fieldstone sample

\section{Conclusion}

$\mathrm{X}$-ray tomography is a powerful tool for the visualization and 3D analysis of geological samples. For the analysis of samples with different components accurate segmentation techniques are necessary. The grains of a Beerlegem fieldstone were segmented using the watershed based separation algorithm. To reduce over-segmentation a smart rejoin technique was applied. Subsequently the glauconite and quartz grains were extracted with the use of different selection parameters. After correct segmentation various parameters of the grains were determined.

\section{Acknowledgements}

The Special Research Fund of the Ghent University (BOF) is acknowledged for the doctoral grant to Loes Brabant. 


\section{References}

BRABANT, L., VLASSENBROECK, J., DE WITTE, Y., CNUDDE, V., BOONE, M.N., DEWANCKELE, J., VAN HOOREBEKE, L. (2011): Three Dimensional Analysis of HighResolution X-ray Computed Tomography Data with Morpho+. Microscopy and Microanalysis, Vol. 17, No. 2. S. $252-263$.

GUAN, W., MA, S. (1998): A List-Processing Approach to Compute Voronoi Diagrams and the Euclidean Distance Transform. IEEE Transactions on Pattern Analysis and Machine Intelligence, Vol. 20, S. 757-761.

OTSU, N. (1979): A threshold selection method from gray-level histograms. IEEE Transactions on Systems, Man and Cybernetics, Vol. 9, S. 62-66.

VINCENT, L., SOILLE, P. (1991): Watershed in digital spaces: an efficient algorithm based on immersion simulations. IEEE Transactions on Pattern Analysis and Machine Intelligence, Vol. 13, S. 583-898.

VLASSENBROECK, J., DIERICK, M., MASSCHAELE, B., CNUDDE, V., VAN HOOREBEKE, L., JACOBS, P. (2007): Software tools for quantification of X-ray microtomography at the UGCT. Nuclear Instruments and Methods in Physics Research Section A: Accelerators, Spectrometers, Detectors and Associated Equipment, Vol. 580, No. 1. S. 442 445. 